\title{
A Bioparticle Separator based on Dielectrophoretic Integrated with Acoustic Force: Design and Simulation
}

\author{
Alireza Dehghani \\ Department of Engineering, \\ Central Tehran Branch, \\ Islamic Azad University, Tehran, Iran
}

\author{
Alireza Kashaniniya \\ Department of Engineering, \\ Central Tehran Branch, \\ Islamic Azad University, Tehran, Iran
}

\begin{abstract}
In this paper, we introduce the application of a microfluidic separator for detection and focusing of two different bioparticles with an integrated method utilizing acoustic and dielectrophoretic (DEP) force. In order to improve separation efficiency, we have integrated two different manipulation techniques. The separator designed and simulated relay on the combination of long-range acoustic waves and short-rang dielectrophoretic forces. The DEP manipulation is generated by an DC or AC non-uniform electronic field, while, acoustic force created using IDTs (Inter Digitated Transducers) patterned on a piezoelectric substrate such as Linbo3 (Lithium Niobate) to generate standing surface acoustic waves. The generated waves move through the PDMS (Polydimethylsiloxane) microchannel located between two same IDTS. We have two different separation steps. In the first step, particles will face with acoustic force to be focused in midline of the microchannel. In the second step, focused particles, will separated with a non-uniform electric field generated by metric 3D electrodes of $\mathrm{Al}$ (Aluminum) mold to the channel wall. These particles will separate and move to the different outlets. First kind of particles have a different manner of the second ones based on differ of their electrical properties. We counted the number of particles in the two different outlets at the end of separating process. Particles numbers showed a well separation efficiency. The method mentioned above is a process that that is enough flexible to utilize in a variety of applications especially in biological purposes.
\end{abstract}

\section{Keywords}

Acoustic force, Dielectrophoresis, BioMEMS, Bioparticle

\section{INTRODUCTION}

Bio-micro electro mechanical systems (Bio-MEMS) are a group of miniaturized microfluidic systems, which have several applications specially in the field of medical diagnostic systems. Cancer is a group of uncontrolled disease which cells of an organ in a body growth unnormally. Early cancer detection is crucial for improved diagnosis and cancer treatment due to the primary tumor Cells size and location [1-4]. There are some common cancer cell detection techniques in microfluidic systems which is based on the difference in physical properties of healthy and cancer cells such as size and density. CTCs (Circulating Tumor Cells) are a group of cancer cells, which are rare in blood sample, especially in the first levels of disease. detection and separation of these rare cells in a few amount of blood sample in patients is an important issue that researchers studying on in the recent years. Most of the microfluidic cancer cell separator focused on cell size, shape and other physical properties. The force utilizing in this biomedical diagnostic systems is based on accuracy and least invasive procedures. Recent technological advances in microfluidic and LOC (LabOn-A-Chip) technology have resulted integrated diagnostic medical micro devices that perform necessary cell manipulation activities like cell pre-filtering, cell fractionation, target cell isolation, cell concentration or focusing, cell lysis, and marker molecule trapping and detection [5]. There is a significant increase of interest in developing techniques that can physically manipulate and separate cells [6,7]. There are different types of forces including optics, acoustic, electric and magnetics that utilize in microfluidic systems. Manipulator based on acoustic force is a non-invasive and long-range force term to move different particles based their size, density and compressibility. Among variety of microfluidic techniques, Acoustophpresis (ACP) and Dielectrophresis (DEP) are lable-free, which affect both physical and electrical properties of particles. The principle of acoustic manipulation relies on acoustic radiation force, which depends on physical properties of particles in an acoustic field generated by acoustic wave to move particles in a desired place inside the microchannel. This method has been used for a long time in macro and micro scaled systems [8-10]. Starting at 2000s, the first step towards miniaturization was taken laminar-flow AC chambers for particle manipulation [11-13] and AC separation in microfluidic (sub-100) capillaries [14]. On the other hand, DEP used for many different applications in Bio-MEMS in the recent years. It is a force due to a non-uniform electric field to push particles and cells from one side to another. Dielectrophresis (DEP) was first described experimentally by Herbert A.Pohl [15,16]. DEP application in biological cells started in 1939 [17]. The DEP force exerted on a cell depends on cell dielectric properties, size, suspending medium properties like permittivity, conductivity and electric field. According to our studies, DEP forces are complementary to acoustic forces. In this study, short-rang dielectrophoretic force combined with long-range acoustophoretic force in a microfluidic separator. Two different steps utilized in sequential procedure, particle focusing and separation. In the first step, particles entered from inlet and focused in midline utilizing acoustic waves generated by IDTs on a piezoelectric substrate and push them to the pressure node made in center of the channel. Then, focused particles are subjected to the non-uniform electric field, the force exerted on the cells move them towards high or low field density regions. Particles subjected in nonuniform electric field polarized and moved to the places of field with higher gradient or lower relay on Clausius-Mossotti factor. This separator system is flexible and applicable for wide range of particles and cells. Typically, acoustic based designs has high throughput in comparison with DEP-based systems. To match the system, three-Dimensional electrodes used to generate electric field inside the 
microchannel. We designed and simulated the separator system based on two kind of different bio-particles focusing on their electrical properties. We proposed that both of the particles have the same size of 8 . Also, permittivity and conductivity of these two particles are $4 . \mathbf{4} \times \mathbf{1 0}^{-\mathbf{1 0}} \mathrm{F} / \mathrm{m}$, $6.6 \times 10^{-10} \mathrm{~F} / \mathrm{m}, 0.5 \mathrm{~S} / \mathrm{m}$ and $0.2 \mathrm{~S} / \mathrm{m}$, respectively. Using standing surface acoustic waves in comparison with bulk acoustic waves has some benefits. Surface acoustic waves generating in the piezoelectric substrate. So, it is stronger than bulk waves which waste their energy in the substrate. We used 3D electrodes attached to the side wall in line of channel height to avoid missing the particles. On the other hand, combining dielectrophoretic with acoustic force results stronger system to trap more particles. As the system is designed for biological applications, and biological particle's properties commonly change by frequency, So, we utilize AC dielectrophresis (AC-DEP) to control particles accurately. The combination of these two methods, dielectrophoresis and acoustophopresis, allowed us for reliable results of variety groups of bio particles. In this paper, we designed and analyzed the system and get the results of COMSOL software.

\section{PRINCIPLES AND THEORY 2.1.AC-DIELECTROPHORETIC MANIPULATION}

DEP is observed when a non-uniform electric field is exerted on the cells because the coulomb forces generated on both sides of the particle are different, thus facilitating the movement of particle towards the region of electric field maxima or minima [18]. There are both DC and AC electric field. Dielectrophresis depends on electrical properties of both particles and medium. Alternating current Dielectrophoresis (AC-DEP) is a technique applying AC voltage to the side wall 3D electrodes. non-uniform generated electric field are used to move bio-particle to different places relay on their permittivity and conductivity. When a bio-particle suspended in a medium, it experiences positive-DEP or negative-DEP. If suspended particles has polarizability higher than the medium, the force will push the particle to the regions of higher electric field gradient (positive DEP). Reversely, if the medium has polarizability higher than particle, it is negative DEP and particle moves toward the places with lower field gradient. The time averaged force on a dielectric particle of radius $r$ in a non-uniform electric field $\mathrm{E}$ can be expressed as follow: [19]

$$
F_{D E P}=2 \pi r^{3} \varepsilon_{m} \operatorname{Re}\left(f_{C M}\right) \nabla E^{2}
$$

$\boldsymbol{\varepsilon}_{\mathbf{p}}{ }^{*}, \boldsymbol{\varepsilon}_{\mathbf{m}}{ }^{*}$ are the complex permittivity of the particle and medium which are related to the conductivity and angular frequency of the electric field.

$$
\varepsilon^{*}=\varepsilon-\mathrm{j} \frac{\sigma}{\varepsilon}
$$

Where is DEP contrast factor (Clausius-Mossotti factor), which for a sphere in an electric field with angular frequency is defined as follow:

$f_{C M}=\frac{\varepsilon^{*} p-\varepsilon^{*} m}{\varepsilon^{*} p+2 \varepsilon^{*} m}$

The indices $\mathrm{p}$ and $\mathrm{m}$ refer of particle and medium, respectively. The sign of the real part of Clausius-Mossotti factor, $\operatorname{Re}\left(f_{C M}\right)$, determines where the particles pushed. If $\operatorname{Re}\left(f_{C M}>0\right)$ Particles move to the places with higher electric field, and vice versa. The transition between negative DEP and positive DEP as particle pushes in AC frequency is known as the cross over frequency. At this frequency, the force experienced by the particle is zero [19]. Cross over frequency rely on the dielectric properties of particles and surrounded medium.

\subsection{PARTICLE MODEL}

Analyzing dielectrophoretic force on biological cells is a challenging issue. Because biological cells have complex and heterogeneous structure, they can not suppose as a simple sphere. So, we have to model bioparticles as single-shell [20,21] or multi-shell particle [22].

In this paper, bioparticles modeled as single-shell, the cell cytoplasm is considered as a sphere covered with a thin shell (cell membrane) and [23] For a single-shell model of a bioparticle, the complex permittivity is given

$\varepsilon_{p}{ }^{*}=\varepsilon_{m} *\left[\frac{\left(\frac{R}{R-d_{\text {mem }}}\right)^{3}+2\left(\frac{\varepsilon_{c y t}{ }^{*}-\varepsilon_{m e m}{ }^{*}}{\varepsilon_{c y t}{ }^{*}-2 \varepsilon_{m e m}{ }^{*}}\right)}{\left(\frac{R}{R-d_{\text {mem }}}\right)^{3}-\left(\frac{\varepsilon_{c y t}{ }^{*}-\varepsilon_{\text {mem }}{ }^{*}}{\varepsilon_{c y t}{ }^{*}-2 \varepsilon_{\text {mem }}{ }^{*}}\right)}\right]$

Where $\mathrm{d}$ is the thickness of the membrane, subscripts cyt and mem represent the cytoplasm and membrane of the cell, respectively [24].

\subsection{STANDING SURFACE ACOUSTIC WAVES MANIPULATION}

SAW-based cell separators mechanism is based on the generation of standing acoustic wave field inside a fluidic microchannel. Suspended particles facing with such acoustic field, experience a primary acoustic force given by: [25]

$F_{r}=-\frac{\left(\pi P^{2} V_{p} \beta_{m}\right)}{2 \lambda} \phi(\beta, \rho) \sin (2 k x)$

$\phi(\beta, \rho)=\frac{\left(5 \rho_{p}-2 \rho_{m}\right)}{\left(2 \rho_{p}+\rho_{m}\right)}-\frac{\beta_{p}}{\beta_{m}}$

where $\mathrm{P}, \mathrm{Vp}, \lambda, \rho, \beta, \mathrm{k}, \mathrm{x}$ are the acoustic pressure amplitude, particle volume, wavelength, density, compressibility, wave number, and the distance from the pressure node respectively. The subscripts of $\mathrm{p}$ and $\mathrm{m}$ denote particle and liquid medium, respectively. When particles are subjected to the acoustic forces, the direction of the particle movement in the SAW field is determined by acoustic contrast factor $(\phi)$ : If $\phi>0$, the particles will be attracted to the pressure node; if $\phi<0$, particles will move to the pressure anti-node. In general, most solid particles including cells suspended in an aqueous medium have positive acoustic contrast factor and they move towards pressure nodes [26]. With comparison of equations expressed by (1) and (5), It appears that DEP force has several similarities with acoustic force. first of all, both of them are related to the size of particle $\left(\propto \boldsymbol{r}^{3}\right)$, and the square of the applied electric potential. On the other hand, contrast factor is the other thing which can be seen in both expressions. Contrast factor in DEP relies on the dielectric properties of particle and surrounding fluid, and in acoustic field expressed by difference in density of particle and medium. 


\section{DESIGN AND SIMULATIONS}

Fig. 1 shows the complete schematic of the separator system.

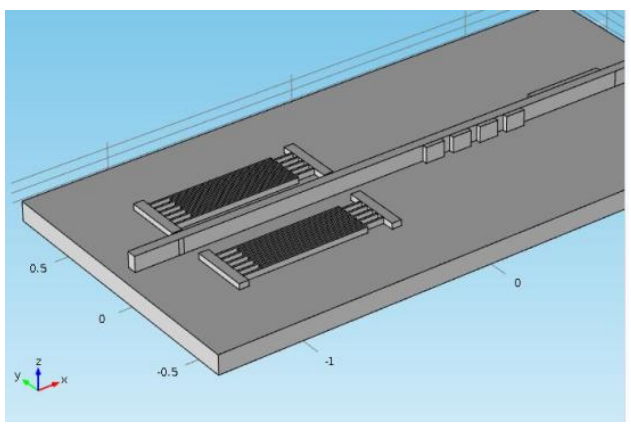

Fig 1. Schematic of the separator system

As mentioned before, the separation process has two steps. In the first part, particles of one inlet enter to the microchannel, LiNbo3 substrate actuated by two Interdigitated Transducer (IDT), to generate surface acoustic waves. Two waves with the same amplitude and 180 degree phase shift, meet each other, generated acoustic force depending on both density and comprehensibility of particles and medium by adjusting the places of IDTs in two sides of the microchannel and pressure node make in the centerline of the microchannel. The length and width of the microchannel in first part are $1000 \mu \mathrm{m}$ and $100 \mu \mathrm{m}$, respectively. Driving frequency $11 \mathrm{MHz}$ and applied voltage is 100 VP-P. As mentioned before, principle of DEP relies on the manipulation of particles in a non-uniform electric field [12]. In this study, the separation step designed by dielectrophoretic force. In the second step, length and width of microchannel set to $60 \mu \mathrm{m}$ and $100 \mu \mathrm{m}$, respectively. One of the benefits of our design is the constant amount of microchannel width. We use seven small electrics in one side and one Large electrode in the other side of the channel. the width of small and large electrodes are 50 and $300 \mu \mathrm{m}$, respectively. The space between small electrodes is $10 \mu \mathrm{m}$. Applied frequency to small electrodes is $1 \mathrm{~Hz}$ and for large electrodes is $100 \mathrm{~Hz}$. Using dual frequency system is beneficial since manner of bioparticles differ in different frequencies, in which one of the targeted particles having negative DEP take to the large electrode and the other particle because of having positive DEP take small electrodes and separated with a well coefficient. Some of the design parameters are listed in Table 1.

Table 1. Parameters value in the separator system

\begin{tabular}{|l|l|}
\hline Parameter & Value \\
\hline Length of microchannel in first part & $1000 \mu \mathrm{m}$ \\
\hline Length of microchannel in second part & $600 \mu \mathrm{m}$ \\
\hline Number of small electrodes & 7 \\
\hline Number of large electrodes & 1 \\
\hline Width of IDTs & $50 \mu \mathrm{m}$ \\
\hline Number of IDTs & 30 \\
\hline
\end{tabular}

\section{SIMULATION RESULTS}

\subsection{ACOUSTIC FORCE SIMULATION}

We utilize COMSOL Multiphysics software. COMSOL analysis is based on finite element method. Because of the complexity of the system design, we use Multiphysics software to couple several physics to obtain situation same as laboratory. At first step, IDTs should be actuated utilizing an ac voltage. Sinusoidal voltage applied on $\mathrm{LiNbO3}$ piezoelectric substrate. IDTs made of Aluminum are periodic in nature consisting of negative and positive potential alternatively. By applying ac voltage with amplitude $100 \mathrm{VP}-\mathrm{P}$ and frequency of $11 \mathrm{MHz}$. piezoelectric substrate actuated sinusoidally. To model this, Piezoelectric Devices (pzd) and electrical circuit(cir) modules of COMSOL utilized. The PDMS microchannel attached to the substrate. To model the generated waves in to the PDMS domain we use solid mechanic module. Elastic material boundary condition in solid module, use to introduce $\mathrm{LiNbO} 3$ and PDMS as isotropic materials. Prescribed displacement utilize for coupling microchannel and substrate domains. We use pzd.uAmpz in $x 3$ direction in our simulation for the boundary between this two area. For pushing particles to move to the pressure nodes it should be generated a pressure node (with the minimum displacement) in midline of the water domain. Pressure acoustic module in frequency domain (acpr), utilize for simulating acoustic pressure field inside the microchannel. In water domain, we solved pressure acoustic module equations, normal acceleration boundary condition with the acceleration equal to acceleration(solid) used to model acoustic pressure field. The figure (1) shown the results of pressure nodes. As mentioned before, by adjusting the places of IDTs, pressure node generated in midline. The yellow part in Fig. 2 shows pressure node.

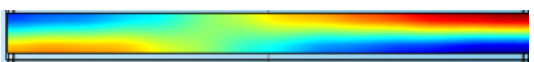

Fig 2. Pressure acoustic field inside the microchannel in first step of separation process

We expected both of the particles move to the center part of the channel in the first step. Creeping flow module (SPF) uses for modeling fluid domain. Inlet and outlet set to normal inflow velocity with the value of and the pressure of zero, respectively. Creeping flow calculated in stationary state. At last, we need to study the particle behavior in fluid domain. Particle tracing for fluid flow (FPT) physics in COMSOL including several forces apply to suspended particles. Applying acoustic force we use acoustophoresis boundary condition as a predefined force in COMSOL. Inlet and outlet of microchannel are set according to the creeping flow. At following, the Figures (3a) to (3e) shown the movement of particles inside the microchannel at different time steps.

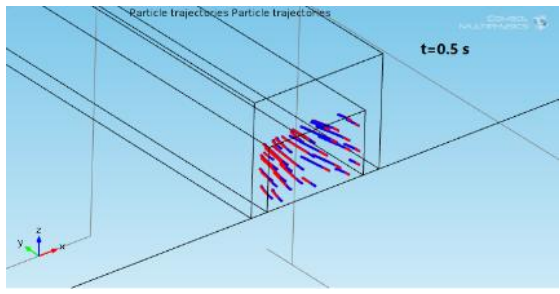

Fig 3a. particle positions at $\mathrm{t}=0$ 


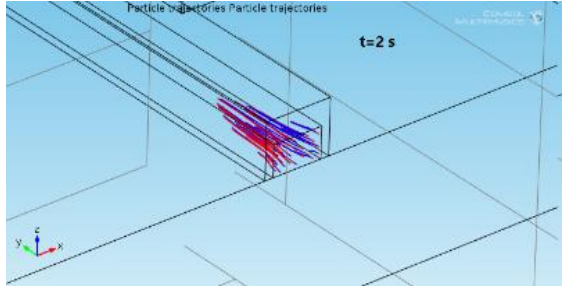

Fig $3 b$. particle positions at $t=2 s$

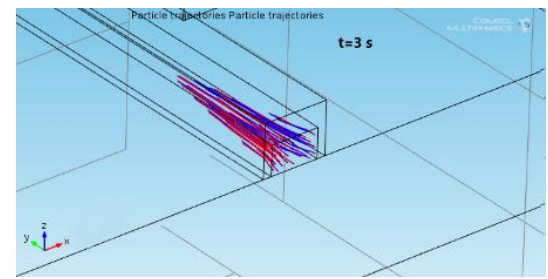

Fig 3c. particle positions at $\mathrm{t}=3 \mathrm{~s}$

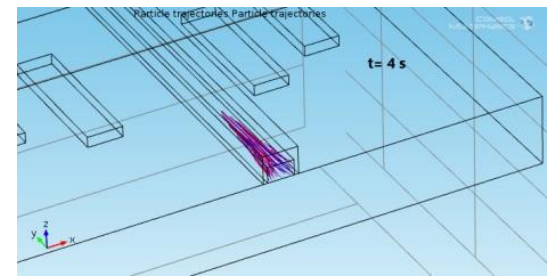

Fig 3d. particle positions at $\mathrm{t}=4 \mathrm{~s}$

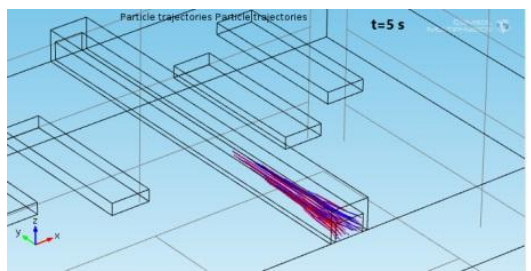

Fig 3e. particle positions at $t=5 \mathrm{~s}$

\subsection{DEP FORCE SIMULATION}

In the second step, focused particles in midline should be separated in a non-uniform electric field. We use seven small electrodes on one side and one large electrode on the other side wall of the microchannel. Since our design based on bioparticle separation we utilize two different frequencies. Applying a voltage with the amplitude of $20 \mathrm{~V}$ and the frequency of $1 \mathrm{~Hz}$ to the small electrodes and $15 \mathrm{~V}$ with the frequency of $100 \mathrm{~Hz}$ o the large electrode we made a non-uniform electric field. Utilizing electric current (EC), creeping flow (SPF) and particle tracing for fluid flow (FPT) we model the second step of the separator system. An alternating voltage of $20 \mathrm{Vp}-\mathrm{p}$ with the frequency of $1 \mathrm{~Hz}$ with the predefined potential boundary condition apply to the small electrodes. Similarity, voltage of $15 \mathrm{Vp}-\mathrm{p}$ with the frequency of $100 \mathrm{~Hz}$ apply to the large electrode with the same boundary condition. Figure 4 shows the electric filed generated inside the channel. As the result, movement of the particles in electric field shown in Figures (5a) to (5f).

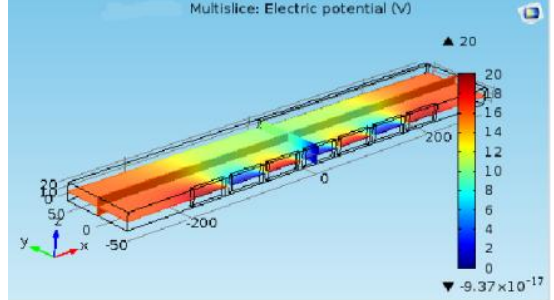

Fig 4. Electric field of COMSOL simulation result

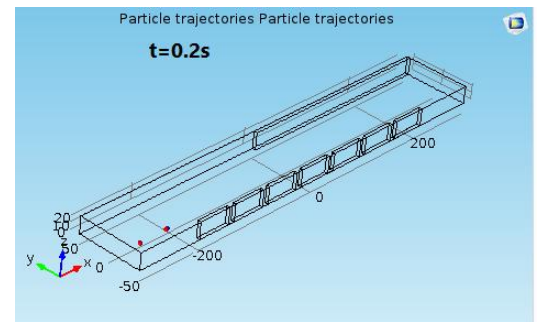

Fig 5a. particles in electric field at $\mathbf{t}=0.2 \mathrm{~s}$

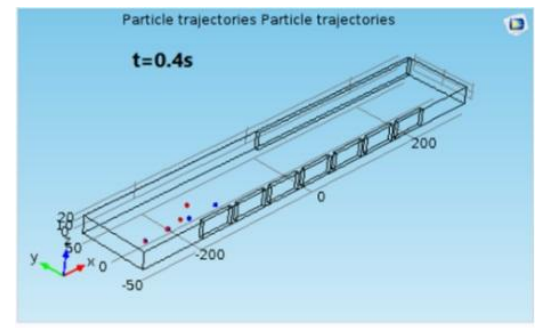

Fig 5b. particles in electric field at $\mathbf{t}=\mathbf{0 . 4 \mathrm { s }}$

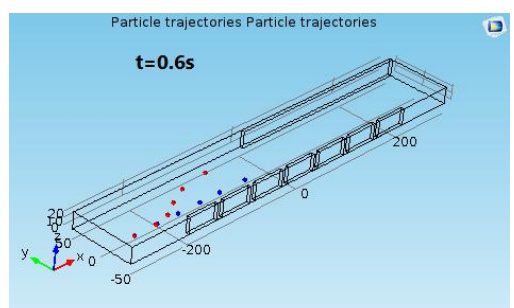

Fig 5c. particles in electric field at $\mathbf{t}=\mathbf{0 . 6 s}$

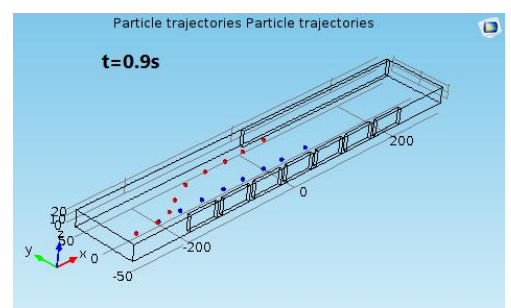

Fig 5d. particles in electric field at $t=0.9 \mathrm{~s}$

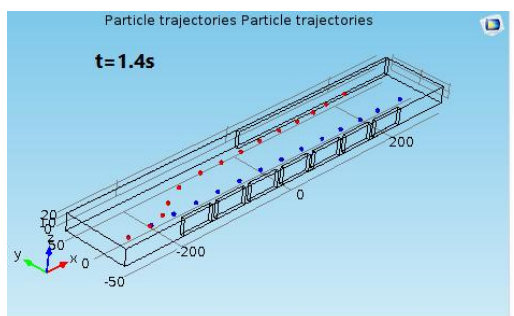

Fig 5e. particles in electric field at $t=1.4 \mathrm{~s}$ 


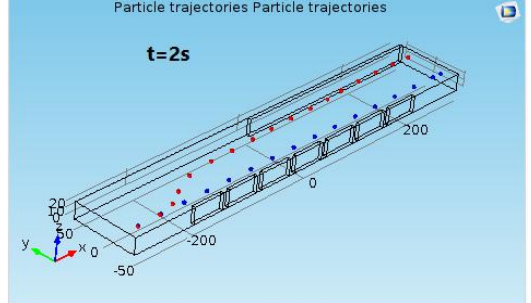

Fig 5f. particles in electric field at $t=2 s$

As mentioned above, particles with negative Clausius-Mossotti factor move to the electric field with low intensity. As shown in the above figures, particles in blue colour because of having negative factor comes down to the microchannel. Reversely, particles in red with positive Clausius-Mossotti factor come up to the top part with electric field with high intensity. The diagram shows in Figure 6a and $6 \mathrm{~b}$, demonstrates the DEP force on red and blue particles versus time.

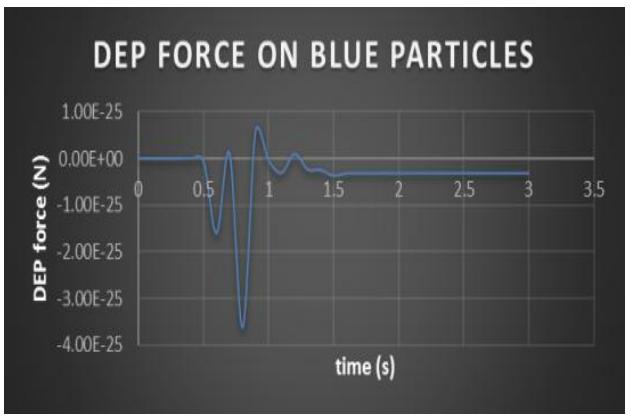

Fig 6a. COMSOL result of DEP force on particles

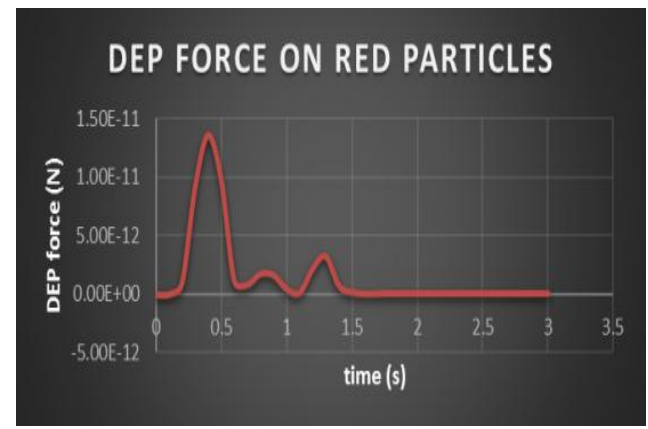

Fig 6b. COMSOL result of DEP force on particles

Particle positions can be followed by the data derived of COMSOL software. Figures $7 \mathrm{a}$ and $7 \mathrm{~b}$ shown the blue and red color particles with negative and positive Clausius-Mossotti factor respectively. As we supposed the midline axes in zero, blue color particles reach the left side wall of the microchannel. Because the electric field intensity is lower near the small electrodes particles with negative factor reach the negative axes point comparing to zero point. Reversely, red color particles take the region with high intensity electric field that is around large electrode area. So these particles go toward the positive points in the axis. As shown in Figure (6a), at first, red particles positioned around - 25 comparing to the axis of midline, then exposing in electric field will reach positive points in the axis. Similarity, blue particles move from -25 to around -21.5 in electric field.

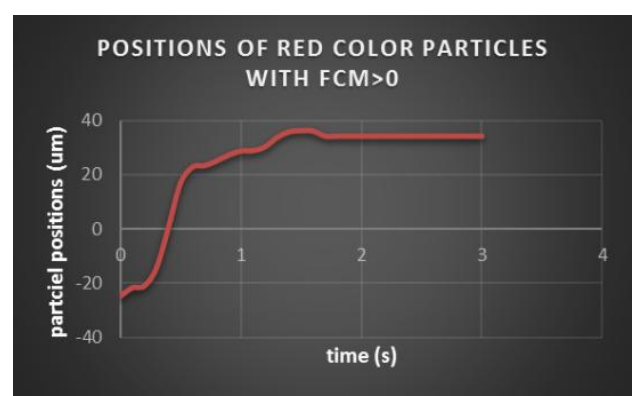

Fig 7a. red color particle positions in electric field versus time

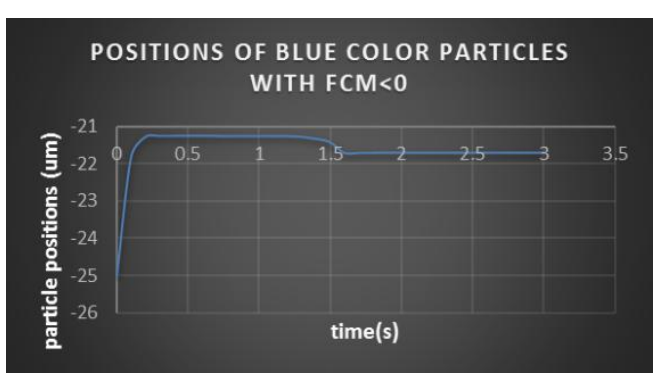

Fig $7 b$. blue color particle positions in electric field versus time

\section{CONCLUSION}

In this paper the theory and principles of using integrated SSAW and DEP force in a separator system are described. The SSAW force affected on the microparticles in first step of separation process is analyzed by finite element model. Red and blue cells with the same size of $8 \mu \mathrm{m}$ reach the pressure node in midline inside the microchannel at the same time exposing acoustic force. Particles focus in midline to avoid dispersion. DEP force in second step affected on particles, red and blue cells have negative and positive clasiuss-mositti of -0.43 and 0.46 , respectively. So, they will be separated successfully. Noted that red and blue particles in simulation process refers to the biological particles with permittivity and conductivity of $4.4 \times 10^{-10} \mathrm{~F} / \mathrm{m}, 6.6 \times 10^{-10} \mathrm{~F} / \mathrm{m}, 0.5 \mathrm{~S} / \mathrm{m}$ and $0.2 \mathrm{~S} / \mathrm{m}$ respectively. The simulation results demonstrated matching between expected and analyzed results.

\section{REFERENCES}

[1] P. Bannasch, Cancer Diagnosis: Early Detection, Springer, Berlin, New York, 1992.

[2] J.M. Elwood and S. B. Sutcliffe, Cancer Control, Oxford University Press, Oxford, New York, 2010.

[3] W. E. O'Donnell, in Early Detection and Diagnosis of Cancer, ed. W.E. O'Donnell, E. Day and L. Venet, C.V. Mosby Co., Saint Louis, 1962.

[4] M. Verma, B. K. Dunn, A. Umar, New York Academy of Sciences and National Cancer Institute (U.S.), Division of Cancer Prevention, Epigenetics in Cancer Prevention: Early Detection and Risk Assessment, New York Academy of Sciences, New York, 2003.

[5] Peter R. C. Gascoyn and Jody V. Vykoukal, Proc IEEE Inst Electr Electron Eng, Dielectrophoresis-Based Sample Handling in General-Purpose Programmable Diagnostic Instruments, 2004 January 1; 92(1): 22-42. 
[6] J. Voldman, Annual Review of Biomedical Engineering, ELECTRICAL FORCES FOR MICROSCALE CELL MANIPULATION, 2006, 8, 425-454.

[7] C.H.Kua, Y.C. Lam,C.Yang and K.Youcef-Toumi, Review of bio-particle manipulation using dielectrophoresis, Singapore-MIT Alliance, 2005.

[8] Nyborg WL. Physical Mechanisms for Biological Effects of Ultrasound.Washington, DC: Department of Health, Education, and Welfare; 1978.

[9] L. A. Crum, Acoustic force on a liquid droplet in an acousticstationary wave, J. Acoust. Soc. Am., 1971, 50, 157-163.

[10] H. M. Hertz, Standing-wave acoustic trap for nonintrusivepositioning of microparticles, J. Appl. Phys., $1995,78,4845-4849$.

[11] J. J. Hawkes, D. Barrow and W. T. Coakley, Microparticle manipulation in millimetre scale ultrasonic standing wave chambers, Ultrasonics, 1998, 36, 925931.

[12] K. Yasuda, Non-destructive, non-contact handling method for biomaterials in micro-chamber by ultrasound, Sens. Actuators, B, 2000, 64, 128-135.

[13] J. J. Hawkes and W. T. Coakley, Force field particle filter, combining ultrasound standing waves and laminar flows, Sens.Actuators, B, 2001, 75, 213-222.

[14] M. Wiklund, S. Nilsson and H. M. Hertz, Ultrasonic trapping in capillaries for trace-amount biomedical analysis, J. Appl. Phys.,2001, 90, 421-426.
[15] H. A. Pohl, Dielectrophoresis the behavior of neutral matter in nonuniform electric fields, Cambridge University Press, Cambridge, 1978.

[16] B. H. Lapizco-Encinas and M. Rito-Palomares, Electrophoresis, 2007, 28, 4521-4538.

[17] G. A. N. Ulrich Zimmermann, 2006.

[18] C. Zhang, K. Khoshmanesh, A. Mitchell and K. Kalantar-zadeh, Anal Bioanal Chem, 2010,396, 401-420.

[19] Nurhaslina Abd Rahman, Fatimah Ibrahim, and Bashar Yafouz, Dielectrophoresis for Biomedical Sciences Applications: A Review, Sensors (Basel). 2017 March; 17(3): 449

[20] Takashima, S., Electrical Properties of Biopolymers and Membranes, Bristol, Adam Hilger 1989.

[21] Irimajiri, A., Hanai, T., Inouye, A., J. Theor. Biol. $1979,78,251-269$

[22] Hu, Q., Joshi, R. P., Beskok, A., J. Appl. Phys. 2009, $106,8,024701(1-8)$

[23] Asami, K., Irimajiri, A.,Biochim. Biophys. Acta 1984, $778,570-578$.

[24] Talukder Z. Jubery, Soumya K. Srivastava, Prashanta Dutta1, Dielectrophoretic separation of bioparticles in microdevices: A review, Electrophoresis 2014, 35, 691713.

[25] Y.K. K. Yosioka, Acoustic radiation pressure on a compressible sphere, Acustica, 5(1955) 167-73.

[26] T. Laurell, F. Petersson, A. Nilsson, Chip integrated strategies for acoustic separation and manipulation of cells and particles, Chem Soc Rev, 36(2007)492-506. 